\title{
ARTICLE
}

\section{$L$-carnitine protects against tacrolimus-induced renal injury by attenuating programmed cell death via PI3K/AKT/PTEN signaling}

Hai-lan Zheng ${ }^{1}$, Hai-yue Zhang ${ }^{2}$, Chun-lian Zhu ${ }^{1}$, Hui-ying Li $^{1}$, Sheng Cui ${ }^{1,3}$, Jian Jin ${ }^{1}$, Shang-guo Piao ${ }^{1}$, Yu-ji Jiang ${ }^{1}$, Mei-ying Xuan ${ }^{1,4}$,

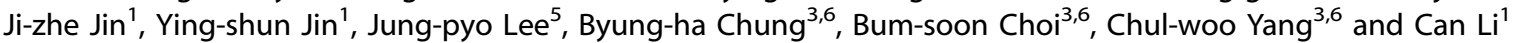

Reducing immunosuppressant-related complications using conventional drugs is an efficient therapeutic strategy. L-carnitine (LC) has been shown to protect against various types of renal injury. In this study, we investigated the renoprotective effects of LC in a rat model of chronic tacrolimus (TAC) nephropathy. SD rats were injected with TAC $\left(1.5 \mathrm{mg} \cdot \mathrm{kg}^{-1} \cdot \mathrm{d}^{-1}, \mathrm{sc}\right)$ for 4 weeks. Renoprotective effects of LC were assessed in terms of renal function, histopathology, oxidative stress, expression of inflammatory and fibrotic cytokines, programmed cell death (pyroptosis, apoptosis, and autophagy), mitochondrial function, and PI3K/AKT/PTEN signaling. Chronic TAC nephropathy was characterized by severe renal dysfunction and typical histological features of chronic nephropathy. At a molecular level, TAC markedly increased the expression of inflammatory and fibrotic cytokines in the kidney, induced oxidative stress, and led to mitochondrial dysfunction and programmed cell death through activation of PI3K/AKT and inhibition of PTEN. Coadministration of LC $\left(200 \mathrm{mg} \cdot \mathrm{kg}^{-1} \cdot \mathrm{d}^{-1}\right.$, ip) caused a prominent improvement in renal function and ameliorated histological changes of kidneys in TAC-treated rats. Furthermore, LC exerted anti-inflammatory and antioxidant effects, prevented mitochondrial dysfunction, and modulated the expression of a series of apoptosis- and autophagy-controlling genes to promote cell survival. Human kidney proximal tubular epithelial cells (HK-2 cells) were treated with TAC $(50 \mu \mathrm{g} / \mathrm{mL})$ in vitro, which induced production of intracellular reactive oxygen species and expression of an array of genes controlling programmed cell death (pyroptosis, apoptosis, and autophagy) through interfering with PI3K/AKT/PTEN signaling. The harmful responses of HK-2 cells to TAC were significantly attenuated by cotreatment with LC and the PI3K inhibitor LY294002 (25 $\mu$ M). In conclusion, LC treatment protects against chronic TAC nephropathy through interfering the PI3K/AKT/PTEN signaling.

Keywords: L-carnitine; tacrolimus nephropathy; oxidative stress; pyroptosis; apoptosis; autophagy; PI3K/AKT/PTEN; HK-2 cells; LY294002

Acta Pharmacologica Sinica (2021) 42:77-87; https://doi.org/10.1038/s41401-020-0449-8

\section{INTRODUCTION}

Tacrolimus (TAC) is a potent immunosuppressive agent that has been widely applied in the management of autoimmune disorders, steroid-resistant nephrotic syndrome, and solid organ transplant rejection. However, the long-term use of TAC is frequently impeded by the emergence of adverse effects, for example, chronic nephropathy, neurotoxicity, infections, malignancies, and new-onset diabetes [1]. Chronic TAC nephropathy reportedly occurs in 5.9\% of people with lupus nephritis [2], 8.3\% of people with steroid-resistant nephrotic syndrome [3], and $22.4 \%$ of kidney transplant recipients [4]. This complication is characterized by proteinuria, progressive renal insufficiency, arteriolopathy, and tubulointerstitial fibrosis (TIF). Although the underlying mechanisms of chronic TAC nephropathy are not yet clear, a complex network mediated by inflammatory cytokines, profibrotic transforming growth factor (TGF)- $\beta 1$, oxidative stress, and programmed cell death may play a role $[5,6]$.

L-Carnitine (LC) is a biologically active stereoisomer of 3-carboxy-2-hydroxy- $N, N, N$-trimethyl-1-propanaminium that functions primarily to transport activated long-chain fatty acids into mitochondria for degradation by $\beta$-oxidation. LC has been shown to inhibit free radical generation, which helps to prevent impairment of fatty acid oxidation in mitochondria and protects tissues from damage by repairing oxidized membrane lipids [7]. Moreover, $\mathrm{LC}$ is considered a direct scavenger of $\mathrm{O}^{2-}$ and $\mathrm{H}_{2} \mathrm{O}_{2}$ [8]. Hence, LC may possess antioxidant properties in addition to its action on lipid metabolism and has been reported to significantly protect against endothelial dysfunction and myocardial infarction [9]. In the kidney, LC attenuates contrast media-induced kidney injury via interference with inflammation and apoptosis [10].

\footnotetext{
${ }^{1}$ Department of Nephrology, Yanbian University Hospital, Yanji 133000, China; ${ }^{2}$ College of Chemical and Life Science, Changchun University of Technology, Changchun 130000, China; ${ }^{3}$ Transplantation Research Center, Department of Internal Medicine, Seoul St. Mary's Hospital, The Catholic University of Korea, Seoul 06591, Korea; ${ }^{4}$ Department of Health Examination Central, Yanbian University, Yanji 133000, China; ${ }^{5}$ Division of Nephrology, Department of Internal Medicine, Seoul National University College of Medicine, Seoul

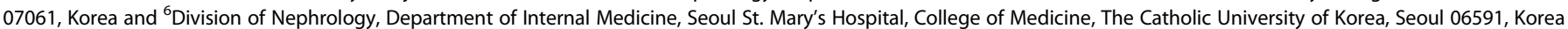
Correspondence: Can Li (lican@ybu.edu.cn)

These authors contributed equally: Hai-lan Zheng, Hai-yue Zhang, Chun-lian Zhu
}

Received: 7 March 2020 Accepted: 21 May 2020

Published online: 17 June 2020 
Similar nephroprotective effects of LC have also been seen in animal models of ischemia-reperfusion (I/R) injury [11], diabetic podocyte injury [12], hypertension-associated renal fibrosis [13], and doxorubicin-induced nephrotic syndrome [14]. However, the possible beneficial effects of LC on TAC-induced renal injury have not been addressed.

Based on the above background information, we hypothesized that LC treatment may confer protection against TAC-induced renal injury. To test this hypothesis, we investigated the effects of LC both in a well-known rat model of chronic TAC nephropathy and in vitro.

\section{MATERIALS AND METHODS}

\section{Materials}

Antibodies specific for the following proteins were used in this study: ectodermal dysplasia-1 (ED-1, Serotec, Oxford, UK), TGF$\beta 1$ (R\&D Systems, Minneapolis, MN, USA), TGF- $\beta$ inducible geneh3 (ßig-h3, Proteintech, Chicago, IL, USA), monocyte chemoattractant protein-1 (MCP-1, Santa Cruz biotechnology, Inc., Delaware Ave, CA, USA), Toll-like receptor-2 (TLR-2, Santa Cruz Biotechnology, Inc., Delaware Ave, CA, USA), interleukin-1 $\beta$ (IL-1 $\beta$, \#ab9722, Abcam, Cambridge, UK), interleukin-18 (IL-18, \#ab191860, Abcam, Cambridge, UK), NOD-like receptor pyrin domain-containing protein 3 (NLRP3, Novus Biologicals, Littleton, CO, USA), 8-hydroxy-2'-deoxyguanosine (8-OHdG, JaICA, Shizuoka, Japan), manganese-containing superoxide dismutase (MnSOD, \#ab13534, Abcam, Cambridge, UK), NADPH oxidase-2 (NOX-2, \#ab31092, Abcam, Cambridge, UK), translocase of the outer mitochondrial membrane 20 (TOMM20, \#ab186734, Abcam, Cambridge, UK), NADH dehydrogenase (ubiquinone) 1 alpha subcomplex 10 (NDUFA10, \#ab96464, Abcam, Cambridge, UK), succinate dehydrogenase complex subunit A (SDHA, \#ab66484, Abcam, Cambridge, UK), dynamin-related protein 1 (DLP1/Drp1, BD Transduction Laboratories, Lexington, KY, USA), optic atrophy protein1 (OPA1, \#ab90857, Abcam, Cambridge, UK), B-cell lymphoma-2 (Bcl-2, \#ab59348, Abcam, Cambridge, UK), Bcl-2-associated X (\#ab32503, Abcam, Cambridge, UK), cleaved caspase-3 (Millipore, Billerica, MA, USA), light chain 3B (\#ab192890, Abcam, Cambridge, UK), Beclin-1 (\#ab62557, Abcam, Cambridge, UK), phosphatase and tensin homolog deleted on chromosome ten (PTEN, Cell Signaling Technology, Danvers, MA, USA), phosphatidylinositol 3-kinase (PI3K, \#ab180967, Abcam, Cambridge, UK), phospho-PI3K (\#ab138364, Abcam, Cambridge, UK), AKT (serine-threonine protein kinase, \#ab8805, Abcam, Cambridge, UK), phospho-AKT (\#ab38449, Abcam, Cambridge, UK), and $\beta$-actin (\#ab8226, Abcam, Cambridge, UK). TAC (Prograf, Astellas Pharma, Ibaraki, Japan) was diluted in olive oil (Sigma, St Louis, MO, USA) to a final concentration of $1.5 \mathrm{mg} / \mathrm{mL}$. LC (Sigma-Aldrich C0158) was diluted in sterile saline to a final concentration of 200 $\mathrm{mg} / \mathrm{mL}$. The doses of TAC and LC used in this study were based on previous reports $[6,15]$.

Experiment and treatment schedule

The experimental protocol was approved by the Animal Care Committee of Yanbian University Hospital (YBU 2018-065). Male Sprague-Dawley rats (Yanbian, China) weighing 210-240 g were housed in individual cages in a temperature- and light-controlled environment and permitted a low-salt diet $(0.05 \%$ sodium, Teklad Premier, Madison, WI, USA) and tap water. Rats were randomly divided into four groups and treated daily for 4 weeks as follows: (1) vehicle group $(\mathrm{VH}, n=8)$ : injected subcutaneously with olive oil $(\mathrm{VH}, 1 \mathrm{~mL} / \mathrm{kg})$; (2) vehicle plus LC group $(\mathrm{VH}+\mathrm{LC}, n=8)$ : treated simultaneously with olive oil and LC $\left(200 \mathrm{mg} \cdot \mathrm{kg}^{-1} \cdot \mathrm{d}^{-1}\right.$, intraperitoneally); (3) TAC group (TAC, $n=8$ ): injected subcutaneously with TAC ( $1.5 \mathrm{mg} / \mathrm{kg}, n=8)$; and (4) TAC plus LC group $(\mathrm{TAC}+\mathrm{LC}, n=8)$ : treated simultaneously with TAC and LC.
Miscellaneous measurements

Rats were pair-fed, and their body weight (BW) was monitored daily. After the 4-week treatment period, rats were housed individually in metabolic cages (Tecniplast, Gazzada, Italy), and their water intake and urine volume were measured over a $24-h$ period. The following day, rats were anesthetized, and blood samples and tissue specimens were collected for further analysis.

Urine protein excretion (UPE) was examined using enzymatic colorimetric methods (Roche Cobas 8000 Core ISE, Roche Diagnostics, Hoffmann-La Roche Ltd., Basel, Switzerland). Markers of renal function, including serum creatinine (Scr), blood urea nitrogen (BUN), and cystatin $C$ (Cys-C) levels were measured by a quantitative enzyme colorimetric method. Systolic blood pressure was recorded in conscious rats by the tail-cuff method with plethysmography using a tail manometer-tachometer system (BP2000, Visitech system, Apex, NC, USA). At least three readings were averaged for each rat. The whole-blood TAC level was measured using LC-MS/MS.

\section{Histopathology}

Kidney specimens were fixed in periodate-lysine-paraformaldehyde solution and embedded in wax. After dewaxing, 4- $\mu$ m sections were processed and subjected to periodic acid-Schiff (PAS) and Masson trichrome staining. Quantitative analyses were performed using a color image autoanalyzer (TDI Scope Eye Version 3.5 for Windows; Olympus, Japan), as we previously described [16].

\section{Immunohistochemistry}

Immunohistochemistry was performed as described previously [6]. Quantitative analyses were performed using a color image analyzer (TDI Scope Eye Version 3.0 for Windows; Olympus, Tokyo, Japan).

\section{Enzyme-linked immunosorbent assay}

Twenty-four-hour urinary concentrations of the DNA adduct 8OHdG were measured using a competitive enzyme-linked immunosorbent assay (Japan Institute for the Control of Aging, Shizuoka, Japan) according to the manufacturer's protocol. All samples were assayed in triplicate.

\section{Transmission electron microscopy}

Transmission electron microscopy was performed as we previously described [17]. Kidney tissues were postfixed with $1 \%$ $\mathrm{OSO}_{4}$ and embedded in Epon 812 following fixation in 2.5\% glutaraldehyde in $0.1 \mathrm{M}$ phosphate buffer. Ultrathin sections were cut and stained with uranyl acetate/lead citrate and imaged with a JEM-1200EX transmission electron microscope (JEOL Ltd., Tokyo, Japan). The number and size of mitochondria in 20 random nonverlapping proximal tubular cells were measured using an automated image analyzer (TDI Scope Eye Version 3.0 for Windows; Olympus, Tokyo, Japan).

\section{Immunoblotting}

Immunoblotting was performed as described previously [18]. Images were analyzed with an image analyzer (Quantity One; BioRad Technical Service Department, Hercules, CA, USA). Optical densities were obtained using the sham group as the reference (set as $100 \%$ ) and normalized to those of $\beta$-actin.

In situ TdT-mediated dUTP-biotin nick-end labeling (TUNEL) assay Apoptosis in tissue sections was identified using an ApopTag in situ Apoptosis Detection Kit (Sigma-Aldrich, Millipore, USA). The number of terminal deoxynucleotidyl transferase-mediated dUTP nick-end labeling (TUNEL)-positive cells was counted in 20 different fields in each section at $\times 400$ magnification. 
Table 1. Effect of LC on basic parameters.

\begin{tabular}{|c|c|c|c|c|}
\hline & $\mathrm{VH}(n=8)$ & $\mathrm{VH}+\mathrm{LC}(n=8)$ & $\operatorname{TAC}(n=8)$ & TAC + LC $(n=8)$ \\
\hline$\triangle B W(g)$ & $45.1 \pm 4.5$ & $46.6 \pm 4.9$ & $17.7 \pm 3.6^{* *}$ & $36.5 \pm 5.8^{\#}$ \\
\hline UV (mL/day) & $9.5 \pm 1.5$ & $10.9 \pm 1.7$ & $16.9 \pm 1.6^{* *}$ & $10.6 \pm 2.3^{\#}$ \\
\hline WI (mL/day) & $31.0 \pm 5.7$ & $35.4 \pm 4.5$ & $37.8 \pm 7.1$ & $32.8 \pm 3.6$ \\
\hline UPE (mg/L) & $305.6 \pm 35.7$ & $298.7 \pm 39.6$ & $582.0 \pm 32.9^{* *}$ & $445.5 \pm 52.3^{\#}$ \\
\hline BUN (mg/dL) & $16.5 \pm 0.5$ & $17.5 \pm 1.7$ & $43.8 \pm 8.0^{* *}$ & $22.8 \pm 3.1^{\#}$ \\
\hline $\mathrm{Scr}(\mathrm{mg} / \mathrm{dL})$ & $0.31 \pm 0.03$ & $0.33 \pm 0.02$ & $0.56 \pm 0.09^{* *}$ & $0.46 \pm 0.05^{\#}$ \\
\hline Cys-C (mg/L) & $2.56 \pm 0.15$ & $2.97 \pm 0.35$ & $4.87 \pm 0.22^{* *}$ & $3.19 \pm 0.34^{\#}$ \\
\hline $\mathrm{SBP}(\mathrm{mmHg})$ & $113.5 \pm 12.3$ & $111.9 \pm 15.1$ & $123.3 \pm 10.8$ & $120.7 \pm 14.5$ \\
\hline TAC con (ng/mL) & ND & ND & $11.8 \pm 1.4$ & $12.2 \pm 1.2$ \\
\hline
\end{tabular}

Cell culture and treatment

Human kidney proximal tubular epithelial cells (HK-2 cells) were obtained from the American Type Culture Collection (Manassas, VA, USA). HK-2 cells were grown in Dulbecco's modified Eagle's medium/Nutrient F12 (HyClone; GE Healthcare Life Science, Logan, UT, USA) supplemented with 10\% fetal bovine serum (Gibco; Thermo Fisher Scientific, Inc., Waltham, MA, USA), $100 \mathrm{U} / \mathrm{mL}$ penicillin, and $100 \mu \mathrm{g} / \mathrm{mL}$ streptomycin (Gibco; Thermo Fisher Scientific, Inc., Waltham, MA, USA). Cells were cultured in a humidified incubator with $5 \% \mathrm{CO}_{2}$ and $37^{\circ} \mathrm{C}$. Cells were pretreated with LC $(100 \mu \mathrm{M})$ at a concentration of $6 \mathrm{pg} / \mathrm{mL}$ for $1 \mathrm{~h}$ and were then treated with TAC $(50 \mu \mathrm{g} / \mathrm{mL})$ or concurrently treated with LC and LY294002 (25 $\mu$ M, SigmaAldrich) for $24 \mathrm{~h}$.

Cell viability assay

HK-2 cell viability was examined using a Cell Counting Kit-8 (CCK8; Dojindo, Kumamoto, Japan) according to the manufacturer's instructions. Approximately $1.0 \times 10^{4}$ cells/well were seeded in a 96-well plate. After treatment with TAC, LC, and LY294002, $10 \mu \mathrm{L}$ of CCK-8 solution was added to each well and incubated at $37^{\circ} \mathrm{C}$ for $3 \mathrm{~h}$. The optical density was calculated from the absorbance measured at $450 \mathrm{~nm}$ (VersaMax Microplate Reader, Molecular Devices, LLC, Sunnyvale, CA, USA).

Measurement of reactive oxygen species (ROS)

The levels of intracellular ROS were measured using $2^{\prime}, 7^{\prime}$ dichlorodihydrofluorescein diacetate (H2-DCFDA; Invitrogen) according to the manufacturer's instructions. HK-2 cells were seeded at a density of $2.0 \times 10^{5}$ cells/well in a six-well plate. After treatment with TAC, LC, and LY294002 for the indicated time, cells were washed with phosphate-buffered saline and were then incubated with $\mathrm{H} 2$ DCFDA for $30 \mathrm{~min}$. Fluorescence was measured using a FACSCalibur flow cytometer (BD Biosciences, San Jose, CA, USA).

Apoptosis assay

Annexin-V-positive HK-2 cells were detected using an Annexin-VFITC apoptosis detection kit (Biosharp, Hefei, China) according to the manufacturer's protocol. Briefly, HK-2 cells were seeded and incubated with TAC $(50 \mu \mathrm{g} / \mathrm{mL})$ with or without LC $(100 \mu \mathrm{M})$ and LY294002 for $24 \mathrm{~h}$, and the samples were analyzed using a FACSCalibur flow cytometer (BD Biosciences, San Jose, CA, USA). The percentage of apoptotic cells was determined relative to the total cell count. The percentage of apoptotic cells was calculated as the number of PI-positive and Annexin-V-positive cells divided by the total number of cells. Three independent experiments were performed.
Statistical analysis

All data are presented as the means \pm SDs. Comparisons among multiple groups were conducted using one-way ANOVA and the Bonferroni post hoc test using SPSS software (version 21.0; IBM, Armonk, NY, USA). Statistical significance was accepted at $P<0.05$.

\section{RESULTS}

Effects of LC on basic parameters

Four weeks of treatment with TAC caused BW loss and polyuria, which were prevented by LC coadministration. Renal dysfunction, as demonstrated by increases in the levels of Scr, BUN, and Cys-C and in UPE in the TAC-treated rats, was significantly improved by coadministration of LC (Table 1), implying a renoprotective effect of LC on chronic TAC nephropathy.

Effects of LC on histopathology

Rats on a low-salt diet that were treated with TAC for 4 weeks showed typical histological features of chronic nephropathy mimicking those observed in humans. PAS and Masson trichrome staining showed that chronic TAC nephropathy manifested as mild glomerular basement thickening, effacement of podocyte foot processes, interstitial inflammatory cell infiltration, tubular vacuolization and atrophy, and striped TIF (Figs. 1a, b and 2a). Quantitative analysis revealed an increase in the fractional mesangial area and TIF in the TAC group, and these pathologies decreased significantly when LC was coadministered. Consistent with this result, the overexpression of TGF- $\beta 1$ and $\beta$ ig-h3 in the TAC group was decreased by LC treatment (Fig. $2 b$ ).

Inflammation plays a critical role in the evolution of chronic TAC nephropathy because it precedes ongoing renal scarring. As shown in Figs. 2 and 3, LC treatment suppressed the upregulation of MCP-1 and TLR-2 expression caused by TAC, and this suppression was accompanied by a decrease in the number of ED-1-positive cells in the glomeruli and interstitium (Fig. 1c, d).

\section{Effects of LC on pyroptosis}

A large body of evidence has demonstrated that pyroptosis is involved not only in acute kidney injury but also in the progression of renal fibrosis in CKD. Therefore, this study examined the expression profiles of pyroptosis-related genes. Our results demonstrated that compared with the VH treatment, long-term TAC treatment significantly increased the expression of IL-1 $\beta$, IL-18, and NLRP3, but that these levels decreased after the addition of LC (Fig. 3). These findings are consistent with the effects of LC on inflammatory parameters observed in this study and indicate an inhibitory effect of LC on pyroptosis. 

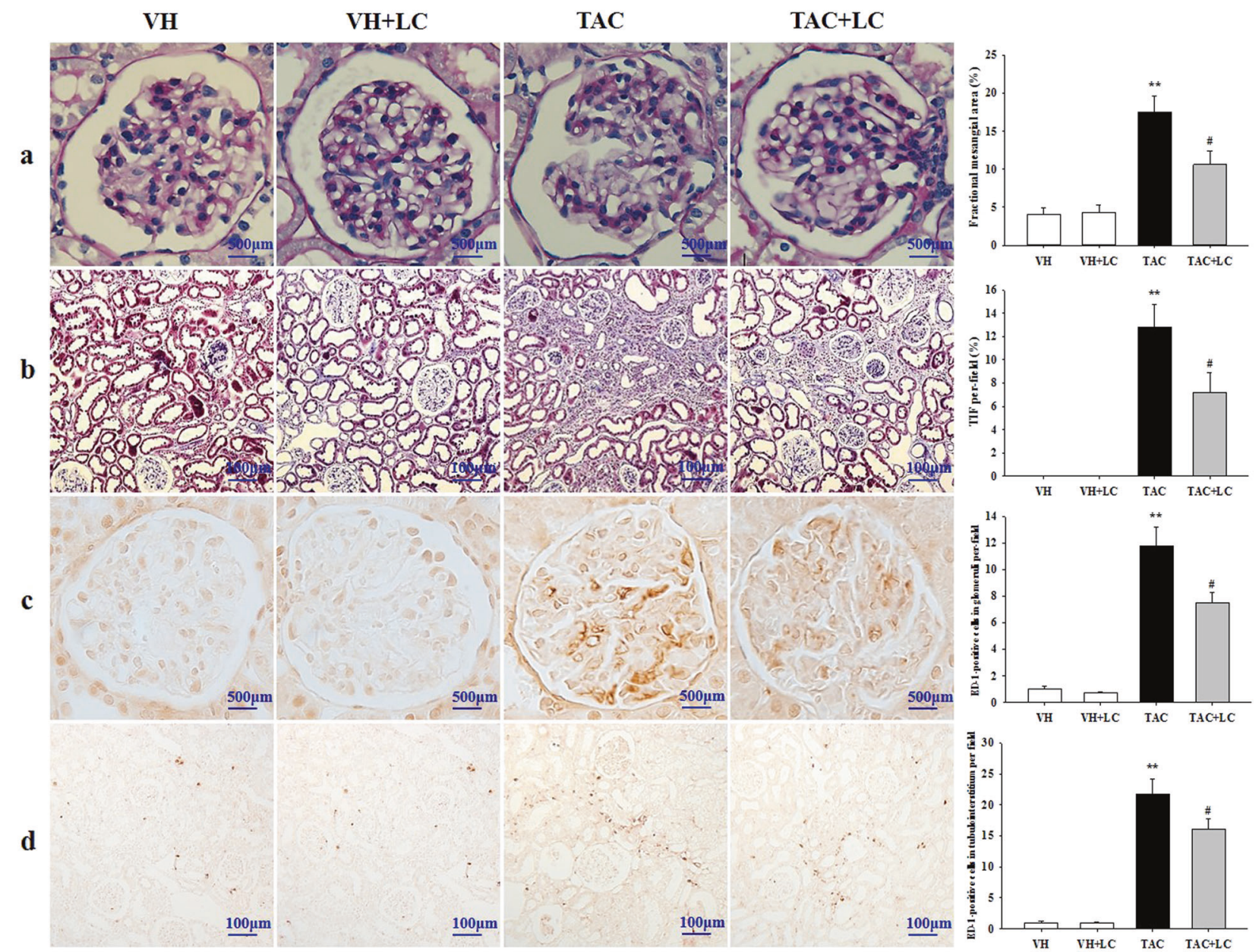

Fig. 1 Representative photomicrographs of PAS (a), Masson trichrome (b), and immunohistochemical staining for ED-1 in glomeruli (c) and the interstitium (d). a, c Original magnification, $\times 1000 ; \mathbf{b}$, d original magnification, $\times 200 ; n=8$. ${ }^{* *} P<0.01$ vs. VH; ${ }^{\#} P<0.05$ vs. TAC.

Effects of LC on oxidative stress

We examined the expression levels of MnSOD, NOX-2, and $8-\mathrm{OHdG}$ in each experimental group. As illustrated in Fig. 4, LC upregulated the expression of the antioxidant MnSOD and downregulated that of NOX-2. In addition, 8-OHdG immunoreactivity and urinary excretion were markedly increased in the TAC group compared with the VH group, whereas LC treatment decreased these levels. This finding confirms the antioxidant capacity of LC in chronic TAC nephropathy, similar to that previously reported by Wang et al. in atherosclerotic myocardium [19] and by Rababa'h et al. in the hippocampus during memory impairment [20].

Effects of LC on mitochondrial dysfunction

By transmission electron microscopy we observed that TAC destroyed mitochondrial structures, as illustrated by disorganized cristae, scattered vacuolization, autophagy formation, mitochondria fragmentation (fission), and mitochondrial fusion (Fig. 5c-f). Quantitative analysis results showed that compared with the TAC treatment, LC treatment restored the number and size of mitochondria. Immunoblotting results revealed that the expression of TOMM20, NDUFA10 (one of the subunits of complex I), and SDHA was significantly suppressed in TACtreated kidneys, but was restored by the addition of LC (Fig. 6). Moreover, dysregulation of proteins related to mitochondrial dynamics (the fission protein DLP1/Drp1 and the fusion protein optic atrophy protein) induced by TAC was balanced by simultaneous administration of LC. These findings suggest that
LC prevents mitochondrial dysfunction in this model of chronic TAC nephropathy.

Effects of LC on apoptosis and autophagy

Emerging evidence has demonstrated that both type I (apoptosis) and type II (autophagy) programmed cell death are involved in the development of chronic TAC nephropathy. Via a TUNEL assay and electron microscopy, we clearly demonstrated that TAC induces apoptotic cell death of renal epithelial tubular cells and interstitial cells and leads to the formation of autophagic compartments, including initial autophagic vacuoles (AVi), degradative autophagic vacuoles (AVd), and autolysosomes (formed from phagophores with multiple- or double-layer membranes) in TAC-treated rat kidneys, while these cell death-related compartments were readily eliminated by LC treatment (Figs. 7 and 8). At the molecular level, LC modulated the expression of a series of apoptosis- and autophagy-controlling genes to promote cell survival. These morphological observations, along with molecular analysis results, indicate that TAC induces excessive apoptosis and autophagic flux, whereas LC treatment reverses these effects.

Effects of LC on the PI3K/AKT/PTEN signaling pathway in vivo To explore the signaling pathway responsible for the renoprotective effects of LC in chronic TAC nephropathy, we used immunoblotting to evaluate signaling through the PI3K/AKT/PTEN axis. The results of this study demonstrated that TAC activated PI3K/AKT although PTEN was silenced and that this pattern was reversed after the addition of LC (Fig. 9). 

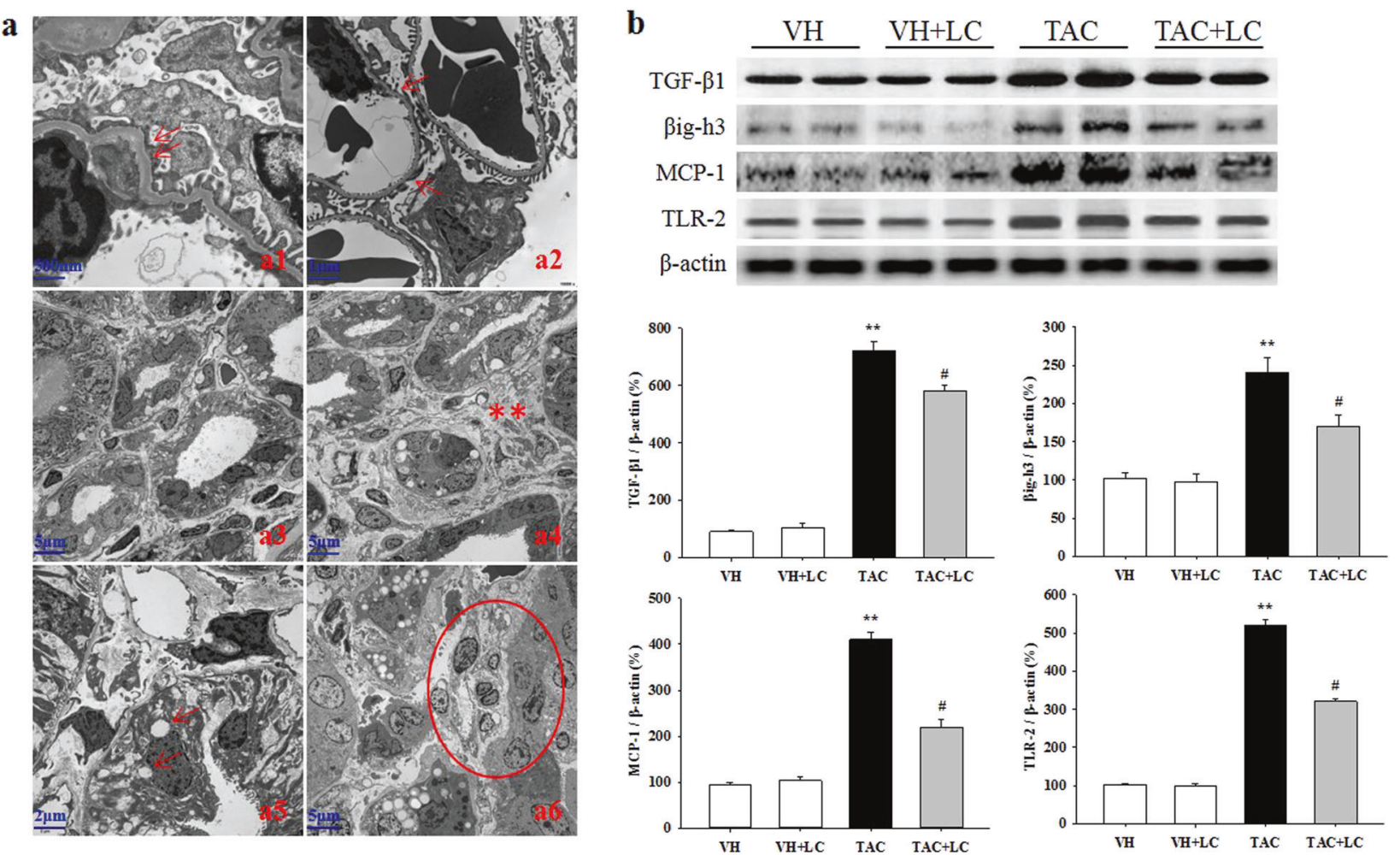

Fig. 2 Representative transmission electron micrographs of TAC-induced renal injury (a) and immunoblot analysis of profibrotic and proinflammatory cytokines in each treatment group (b). a1: mild glomerular basement membrane thickening (arrows, $\times 20,000$ ); a2: effacement of podocyte foot processes (arrows, $\times 10,000)$; a3: tubular atrophy $(\times 2500)$; a4: tubulointerstitial expansion and collagen deposition (star-like symbols, $\times 2500$ ); a5: tubular vacuolization (arrows, $\times 5000$ ); a6: inflammatory cell infiltration within the tubulointerstitium (circle, $\times 2500) ; n=8$. ${ }^{* *} P<0.01$ vs. $\mathrm{VH} ;{ }^{\#} P<0.05$ vs. TAC.

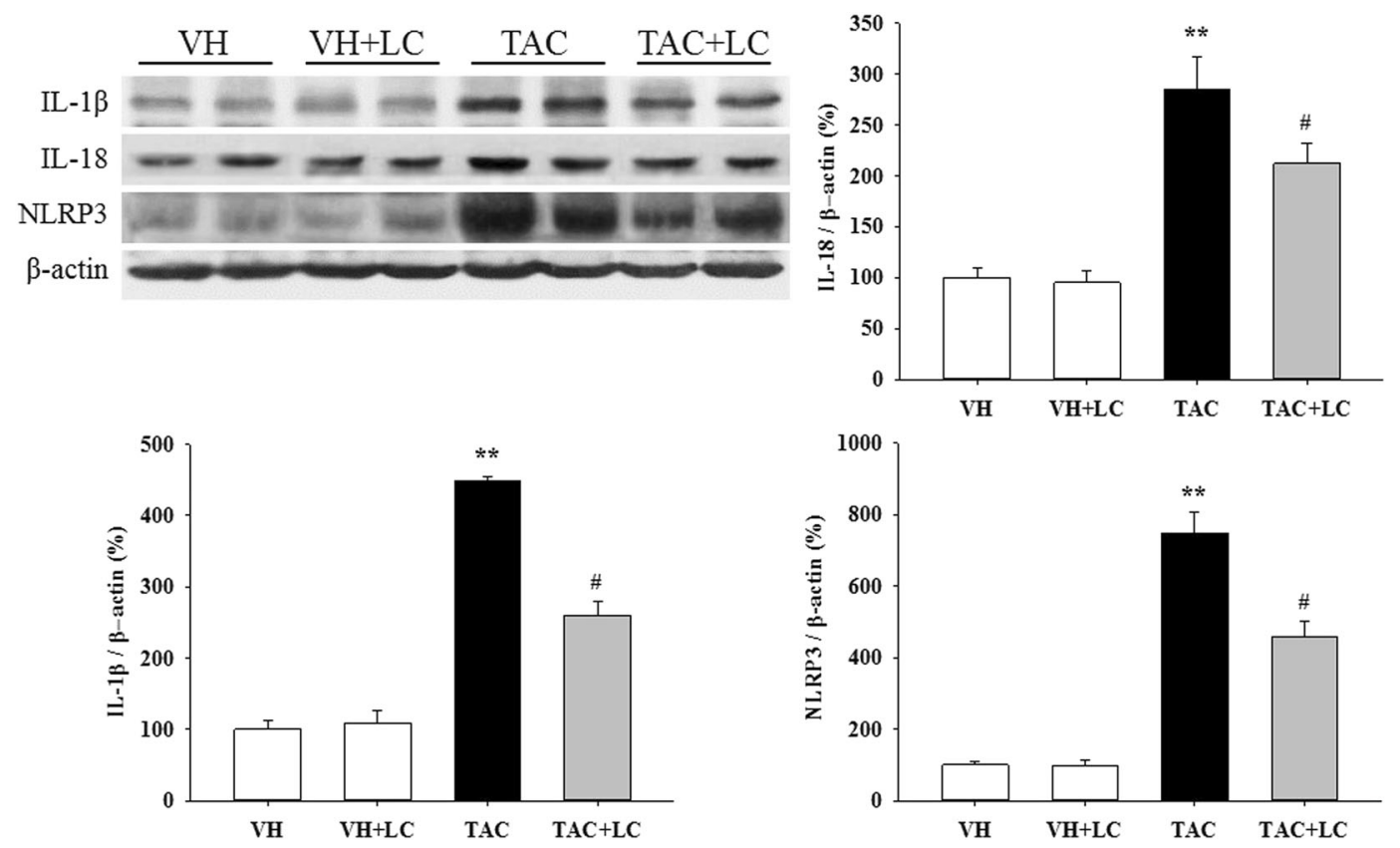

Fig. 3 Representative photomicrographs of the immunoblot analysis of pyroptosis-related genes in each treatment group; $n=8$. ${ }^{* *} P<0.01$ vs. $\mathrm{VH} ;{ }^{\#} P<0.05$ vs. TAC.

Effect of LC and LY294002 on TAC-treated HK-2 cells

To substantiate the effects of LC in the rat model of chronic TAC nephropathy, we performed a concomitant in vitro study in which HK-2 cells were subjected to TAC treatment in the presence or absence of LC and LY294002. In parallel with the results of the in vivo study, LC inhibited intracellular ROS production, and upregulated MnSOD expression but downregulated NOX-2 expression. Suppression of oxidative stress by LC was associated with a 


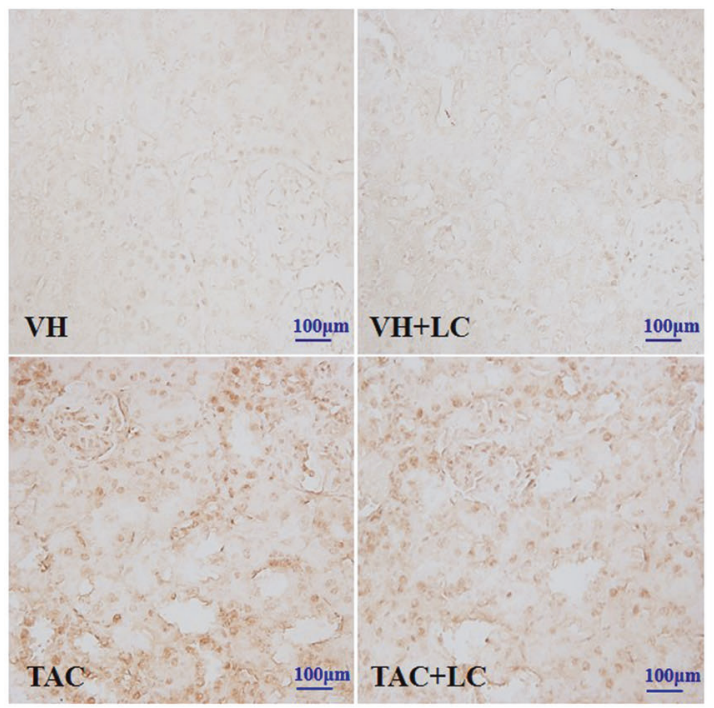

b

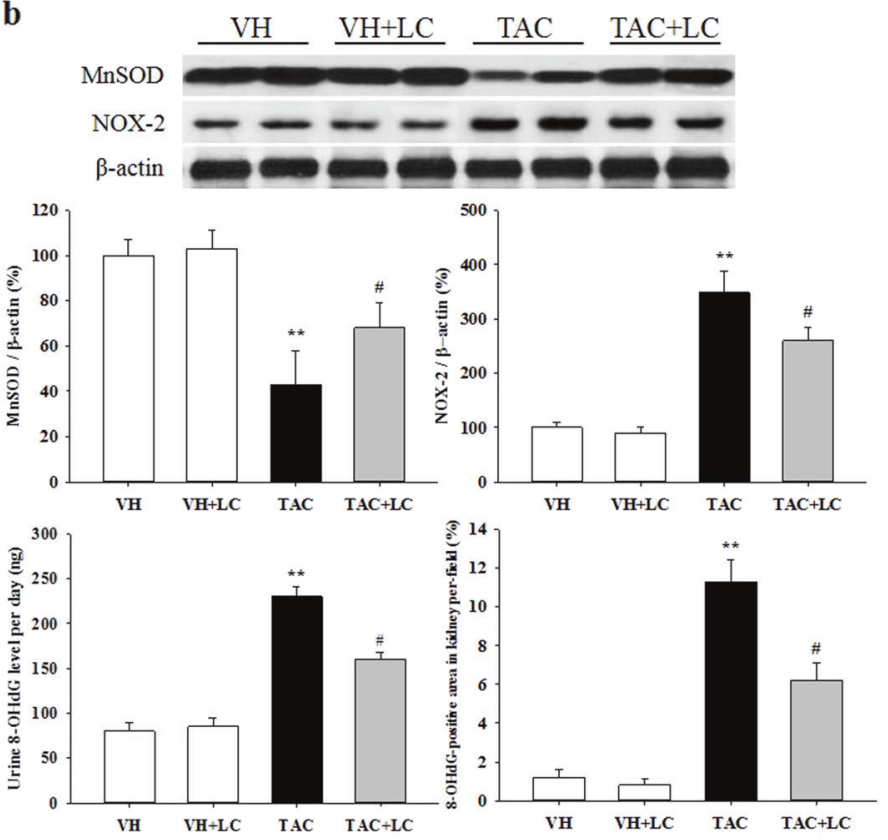

Fig. 4 Representative photomicrographs of immunohistochemical staining of 8-OHdG (a), urine 8-OHdG excretion, and immunoblot analysis of the oxidant NOX-2 and antioxidant MnSOD enzymes in each treatment group (b). Immunohistochemical staining, original magnification, $\times 200 ; n=8$. ${ }^{* *} P<0.01$ vs. $\mathrm{VH} ;{ }^{\#} P<0.05$ vs. TAC.
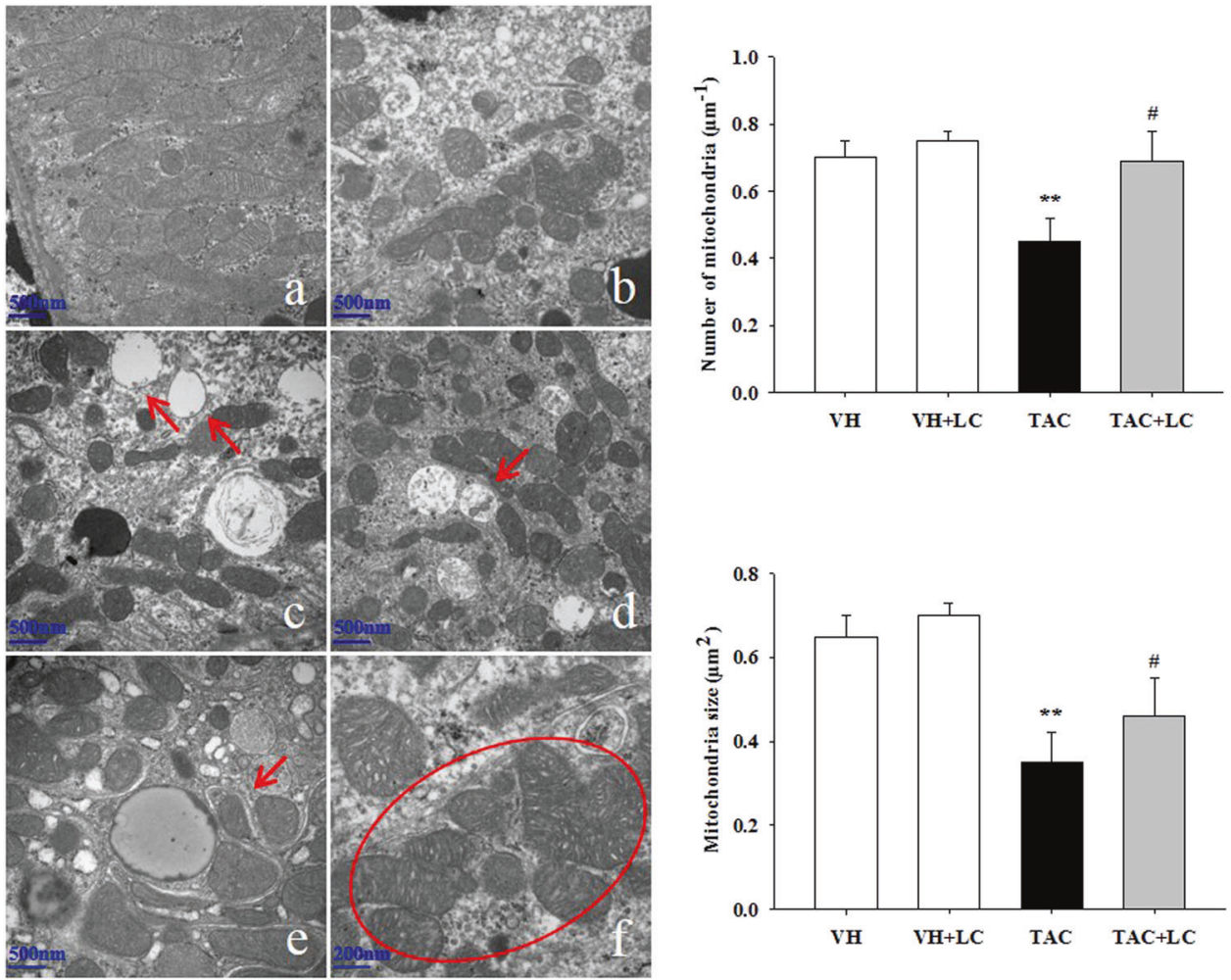

Fig. 5 Representative transmission electron micrographs of mitochondrial morphology and quantitative analysis of mitochondrial number and size in each treatment group. a Normal mitochondria $(\times 20,000)$; $\mathbf{b}$ reduced mitochondrial number in the kidneys of TAC-treated rats $(\times 20,000)$; c mitochondrial vacuolization (arrows, $\times 20,000)$; d autophagy formation (arrow, $\times 20,000$ ); e a mitochondrion divided into two daughter organelles (fission; arrow, $\times 30,000$ ); $\mathbf{f}$ mitochondrial fusion (circle, $\times 40,000$ ); $n=8 .{ }^{* *} P<0.01$ vs. VH; ${ }^{\#} P<0.05$ vs. TAC.

reduction in HK-2 cell death and the regulation of genes controlling programmed cell death (pyroptosis, apoptosis, and autophagy) through interference with PI3K/AKT/PTEN signaling, effects that were enhanced by the PI3K inhibitor LY294002 (Figs. 10 and 11).

\section{DISCUSSION}

LC supplementation is usually applied to manage carnitine deficiency in patients on dialysis. Carnitine deficiency resulting from genetic disorders, disruption of carnitine intake and 

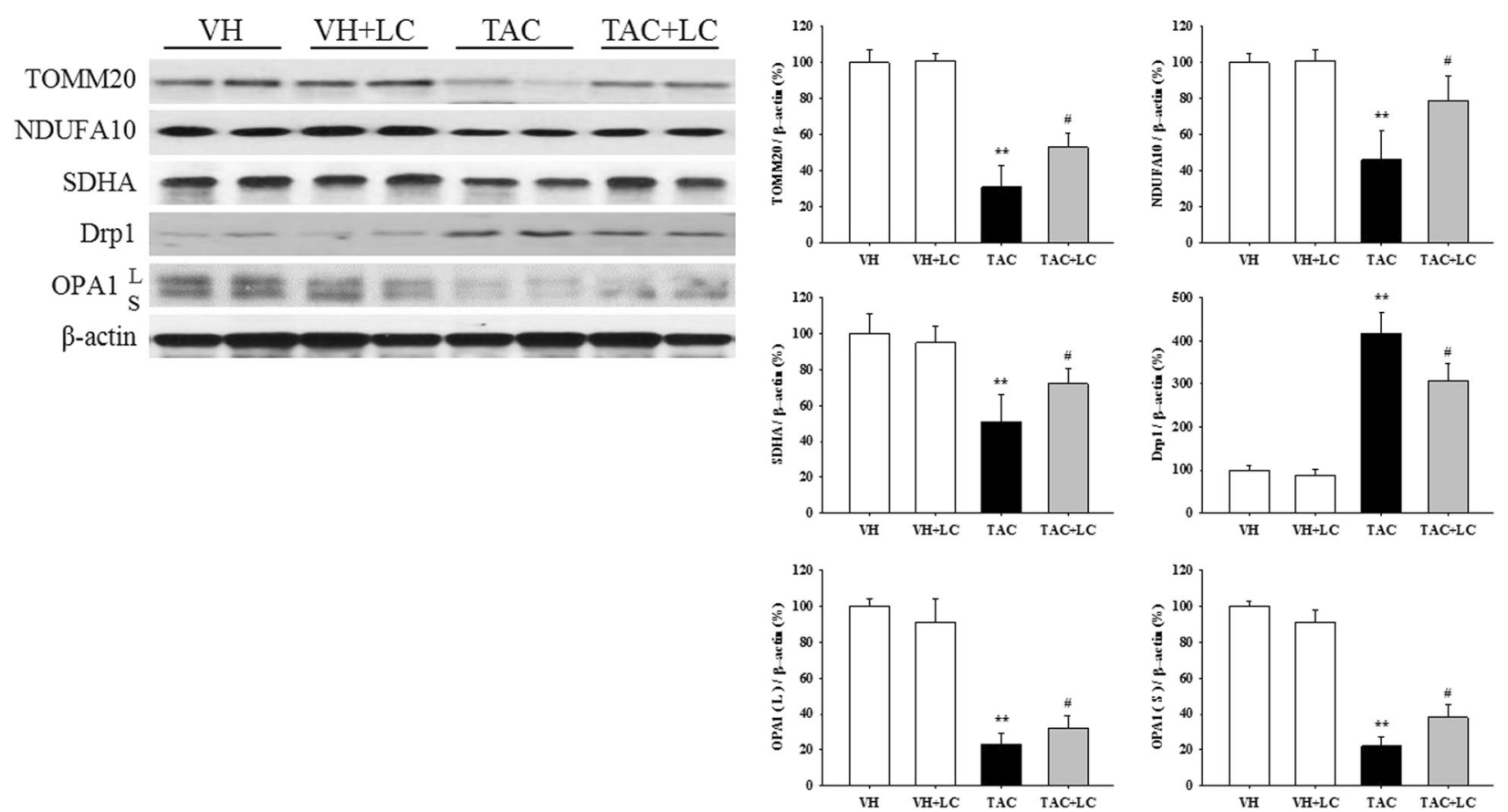

Fig. 6 Representative photomicrographs of immunoblot analysis of proteins related to mitochondrial function and dynamics; $n=8$. ${ }^{* *} P<0.01$ vs. VH; ${ }^{\#} P<0.05$ vs. TAC.

a
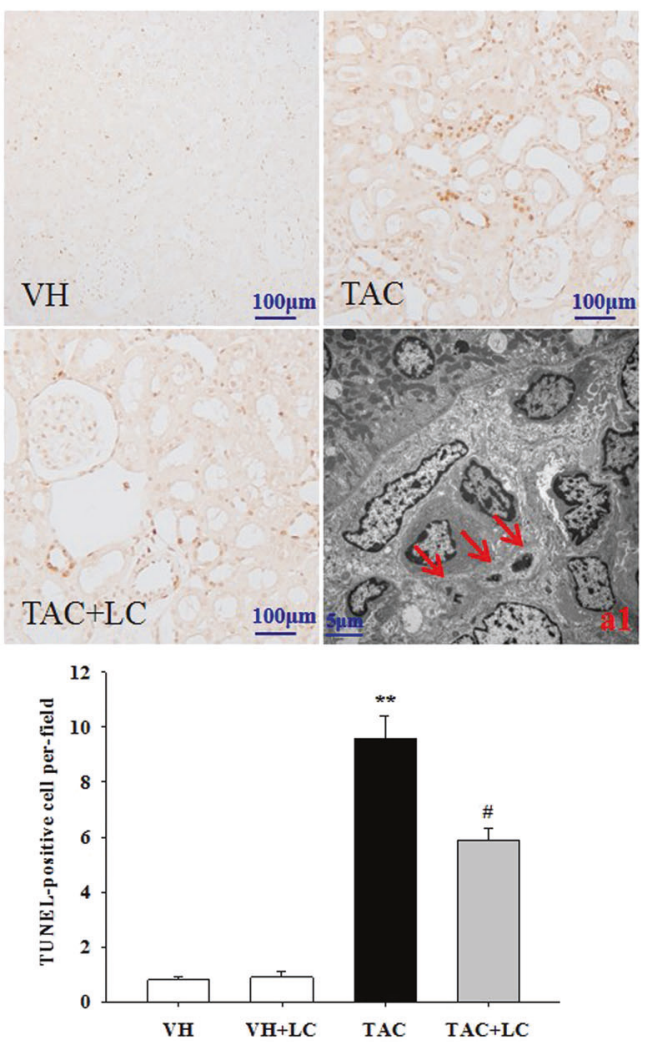

b

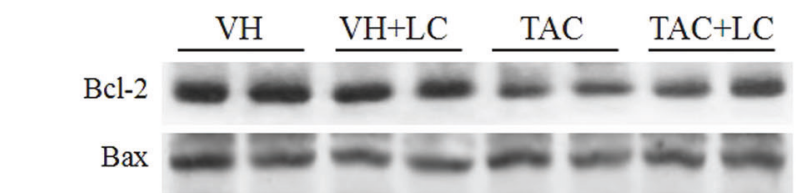

Cleaved

caspase-3

$\beta$-actin
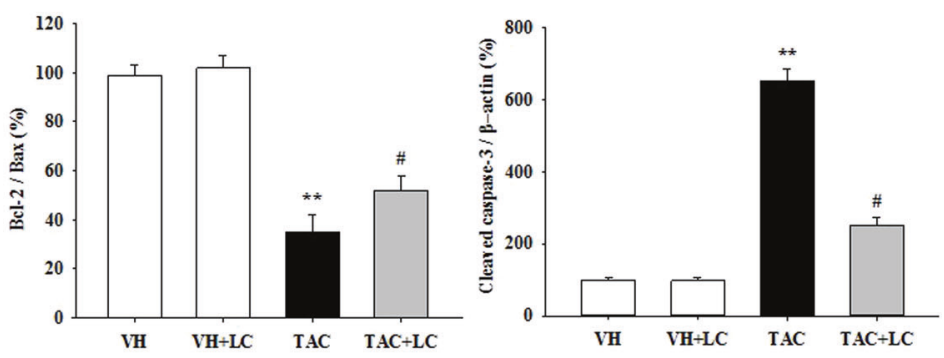

Fig. 7 Representative photomicrographs of the TUNEL assay results (a) and transmission electron micrographs (a1) and photomicrographs of the immunoblot analysis of apoptosis-related genes (b). TUNEL assay, original magnification, $\times 200 ;$ a1: apoptotic bodies (arrows, $\times 2500$ ); $n=8$. ${ }^{* *} P<0.01$ vs. VH; ${ }^{\#} P<0.05$ vs. TAC. 

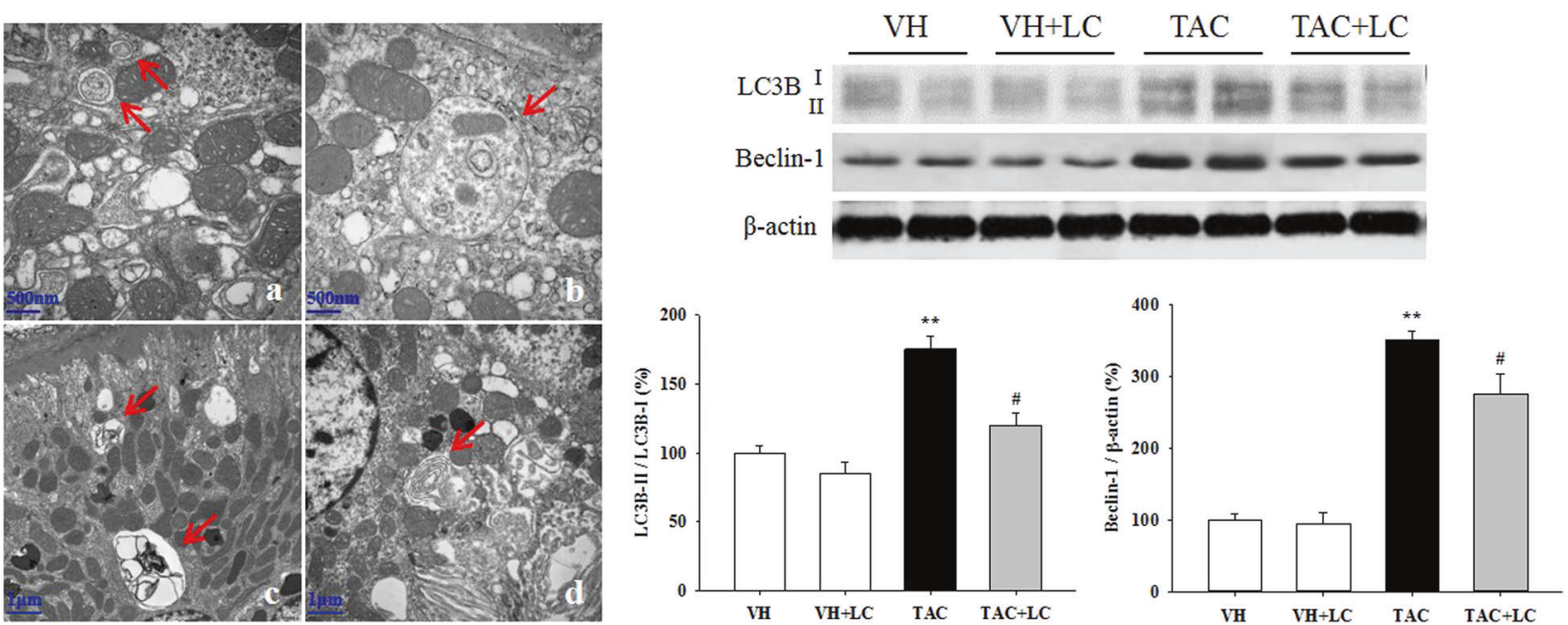

Fig. 8 Representative transmission electron micrographs of autophagic compartments and photomicrographs of the immunoblot analysis of autophagy-related genes. a initial autophagic vacuoles (AVi; arrows, $\times 30,000$ ); b AVi containing mitochondria (arrow, $\times 30,000$ ); c degradative autophagic vacuoles (AVd; arrows, $\times 10,000)$; d autolysosome, formed from phagophores with multiple- or double-layer membranes (arrow, $\times 15,000) ; n=8 .{ }^{* *} P<0.01$ vs. $\mathrm{VH} ;{ }^{\#} P<0.05$ vs. TAC.
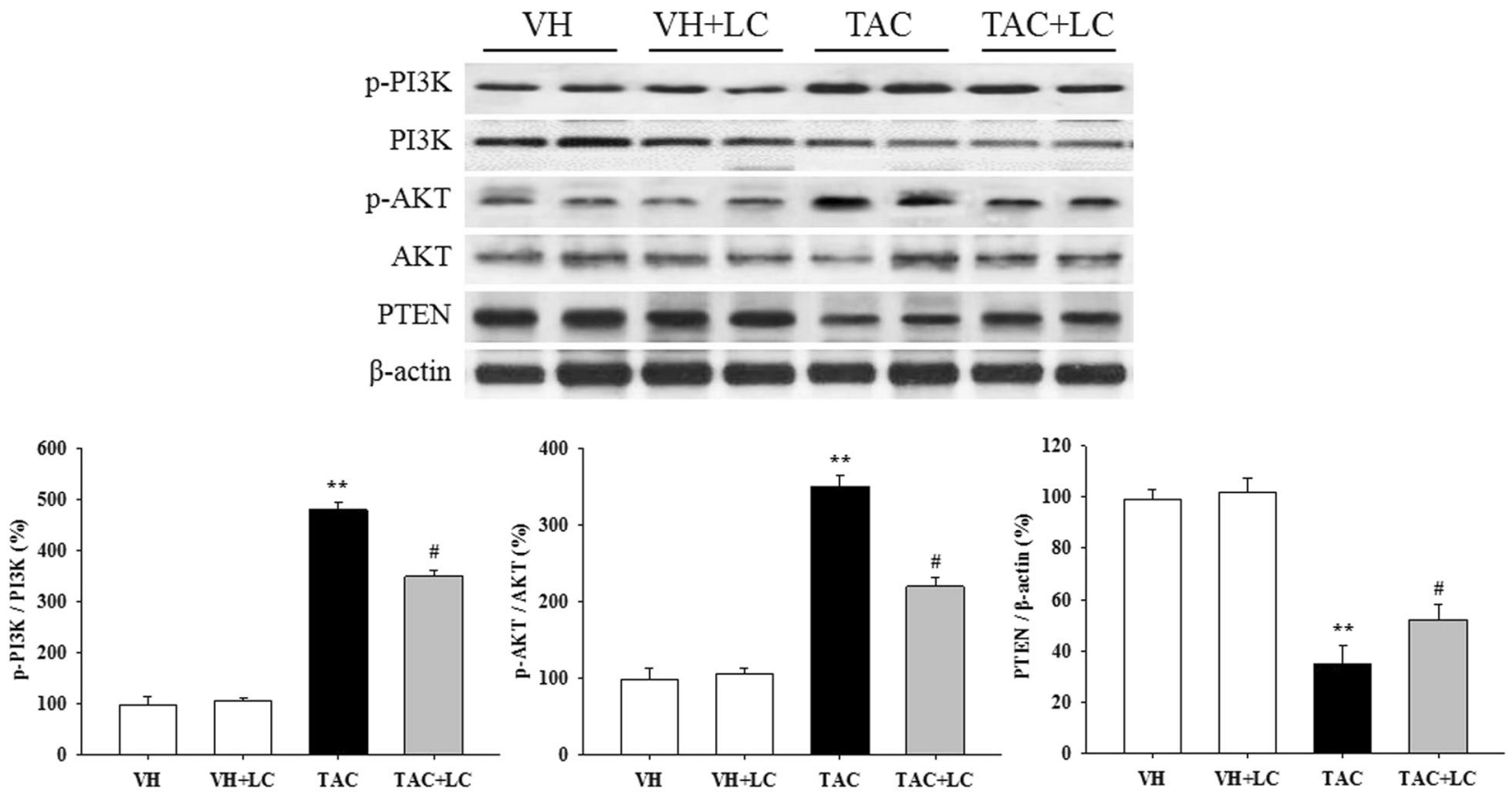

Fig. 9 Representative photomicrographs of the immunoblot analysis of PI3K/AKT/PTEN signaling; $n=8$. ${ }^{*} P<0.01$ vs. VH; ${ }^{*} P<0.05$ vs. TAC.

biosynthesis or removal of carnitine by hemodialysis is associated with numerous pathological conditions, including cardiac dysfunction, muscle weakness, and erythropoietin-resistant anemia. However, LC possesses antioxidative and anti-inflammatory properties and may play diverse roles. $\mathrm{Li}$ et al. reported that LC attenuates myocardial injury after cardiopulmonary bypass in patients with rheumatic valvular heart disease by mitigating inflammatory cytokine production and modulating oxidant enzymes [21]. The benefits of LC have also been observed in fatty liver disease [22], metabolic disorders [23], and polycystic ovary syndrome [24]. The results of the present study showed that LC treatment reduces urinary protein excretion and improves renal function and histopathology. We propose that administering LC to CKD patients as early as possible may result in extra benefits beyond those of carnitine supplementation alone. Further clinical trials are needed to test this hypothesis.
The mechanism responsible for the renoprotective effects of LC in this model may be multifactorial, and may involve a reduction in inflammation. Recently, it was shown that the NLRP3 inflammasome triggers inflammation via inflammasome-dependent and/or inflammasome-independent pathways via a series of biological processes, including cleavage of procaspase- 1 , activation and maturation of gasdermin $D$, and release of IL-1 $\beta$ and IL-18, leading to pyroptosis [25]. Pyroptosis, a unique type of programmed cell death distinct from apoptosis, has been demonstrated to participate in acute kidney injury [26], unilateral ureteral obstruction (UUO) [27], lupus nephritis [28], and diabetic kidney disease [29]. NLRP3knockout mice showed less fibrosis, apoptosis, and ROS-induced injury than wild-type mice [30]. Moreover, LC reduces inflammation in hemodialysis patients with hyperlipoproteinemia [31] and in animal models of hypertension-associated renal fibrosis [13]. In this study, we observed that TAC treatment significantly upregulated the 

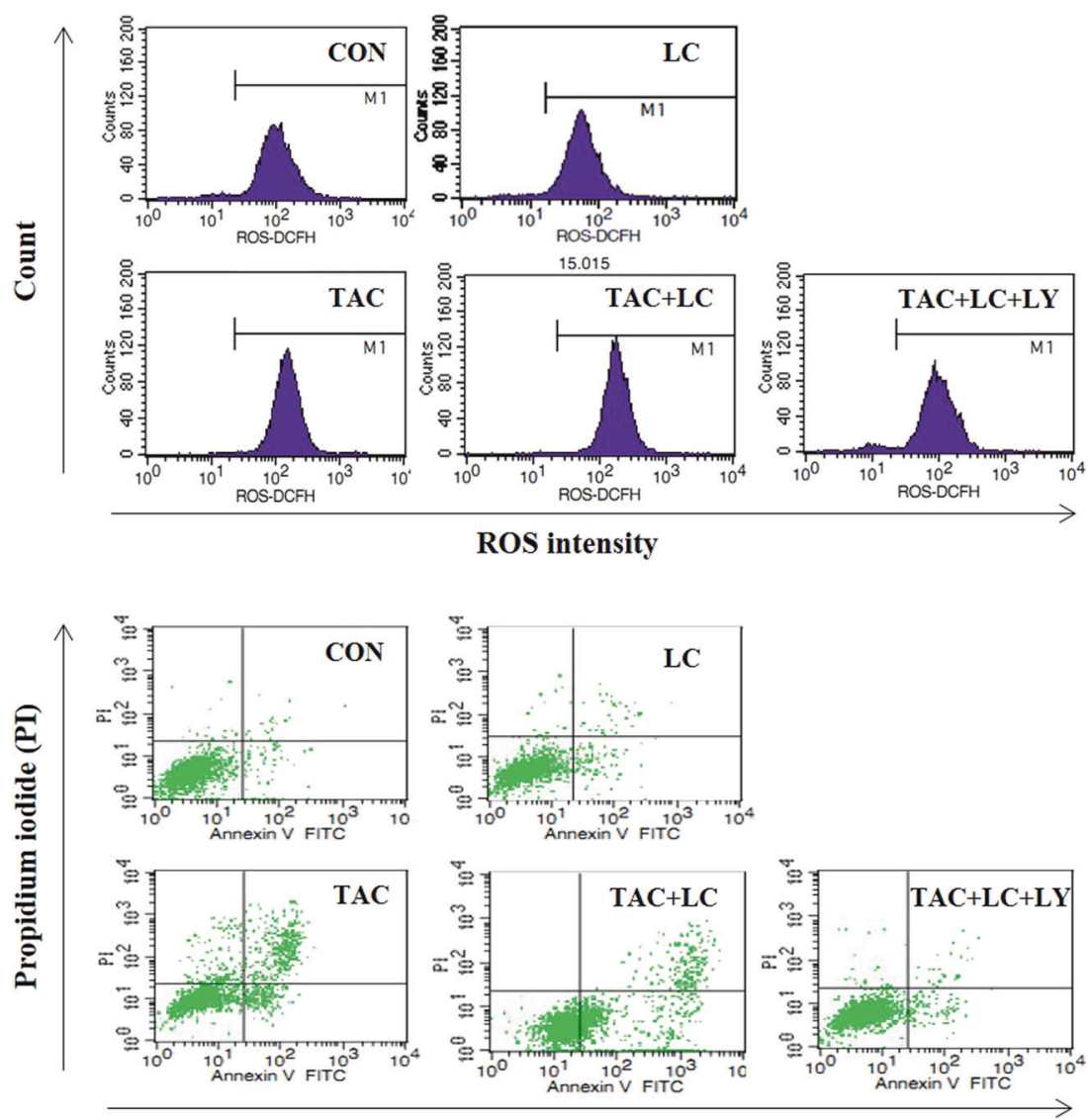

Annexin-V/FITC
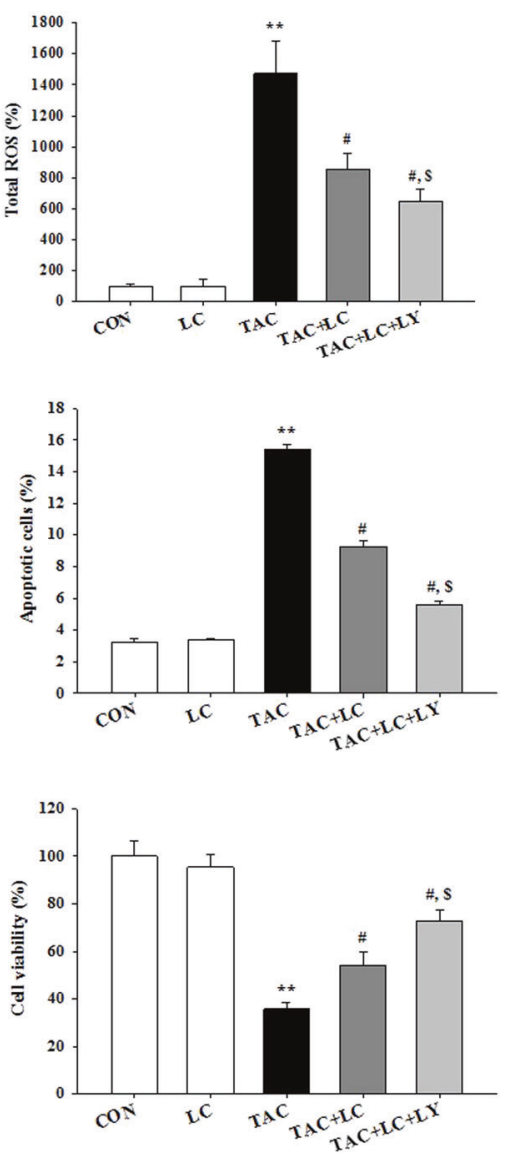

Fig. 10 Effect of LC and LY294002 on intracellular ROS production; apoptosis assay and CCK-8 cell viability assay of TAC-treated HK-2 cells; $n=$ 3. ${ }^{* *} P<0.01$ vs. VH; ${ }^{\#} P<0.05$ vs. TAC; ${ }^{\$} P<0.05$ vs. TAC $+\mathrm{LC}$.

expression of pyroptosis-controlling genes (NLRP3, IL-1 $\beta$, and IL-18), accompanied by an increase in ED-1-positive cells and proinflammatory cytokines, all of which were abolished by the addition of LC. These findings raise the possibility that the anti-inflammatory effects of LC in this model may be attributable to its action on pyroptosis.

It is also possible that the renoprotective effects of LC in this model may be related to its action on oxidative stress-induced mitochondrial dysfunction. Mitochondria are highly plastic organelles involved in macromolecule biosynthesis, energy homeostasis, oxidative phosphorylation, inflammation, and cell death [32]. As a result, mitochondria are highly susceptible to oxidative stress in the kidney because of their high oxygen consumption and abundance. We and others demonstrated previously that TAC facilitates mitochondrial dysfunction via oxidative stress and that LC, an endogenous mitochondrial membrane compound, prevents mitochondrial dysfunction via its antioxidant properties $[17,33,34]$. In the present study, we found that LC treatment restored the mitochondrial number and size and modulated the expression of a number of genes controlling mitochondrial dynamics and function, and we observed that these effects were associated with reduced oxidative stress. Thus, it appears that elimination of oxidative stress and preservation of mitochondrial fitness by LC may account for its renoprotective effects.

Regardless of the role of pyroptosis, both apoptosis (type I programmed cell death) and autophagy (type II programmed cell death) are involved in the pathogenesis of chronic TAC nephropathy. We recently showed that long-term TAC treatment directly induces the apoptosis and autophagy of renal tubular epithelial cell or indirectly induces these types of programmed cell death via oxidative stress or TGF- $\beta 1[18,35]$. Thus, TAC-induced oxidative stress and programmed cell death work in concert to exacerbate renal injury. Moreover, LC protects against carboplatin- or cyclosporine-induced renal injury by inhibiting apoptotic cell death $[15,36]$. Via a TUNEL assay and electron microscopy, we clearly observed that LC significantly mitigated TAC-mediated increases in the number of TUNEL-positive cells and the formation of autophagic compartments (e.g., AVi, AVd, and autolysosomes). In both the rat model of chronic TAC nephropathy and in HK-2 cells treated with $\mathrm{LC}$, these morphological changes were accompanied by regulation of a number of apoptosis- or autophagy-controlling genes to promote cell survival. It seems that LC modulates programmed cell death in a manner suggesting its potential for the treatment of fibrosis in chronic TAC nephropathy.

The PI3K/AKT pathway is an intracellular signaling pathway of particular importance in cell quiescence, proliferation, cancer, and longevity. Activation of PI3K phosphorylates and further stimulates AKT localized in the cell membrane, and activated AKT triggers various biological functions. By contrast, this pathway is antagonized by tensin homolog (PTEN). Evidence has emerged that the PI3K/AKT/PTEN signaling pathway is involved in the pathogenesis of numerous kidney diseases, including renal $I / R$ injury [37], UUO [38], and diabetic nephropathy [39]. In this study, we found that LC treatment abrogated the PI3K/AKT activation and PTEN inhibition caused by TAC and that this effect was enhanced by treatment with a PI3K inhibitor. Moreover, LC increased the level of the antioxidant protein MnSOD, thereby inhibiting programmed cell death in rat kidneys and HK-2 cells. Indeed, the PI3K/AKT/PTEN signaling axis links oxidative stress with programmed cell death; thus, LC counteracts oxidative stressmediated programmed cell death.

In summary, this study demonstrated that LC is capable of attenuating TAC-induced renal injury. Inhibition of oxidative 


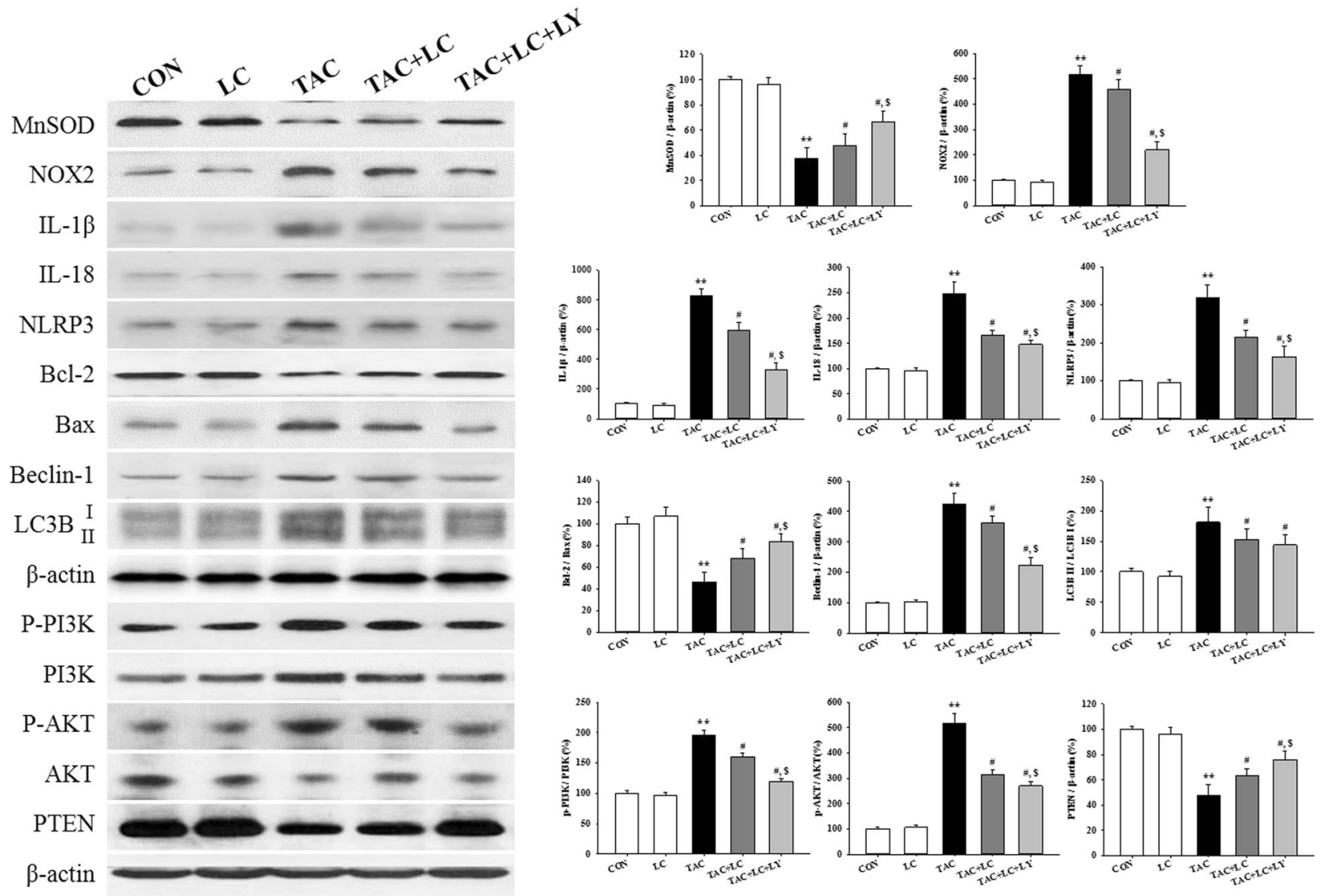

Fig. 11 Effect of LC and LY294002 on oxidative stress enzymes, a range of programmed cell death-controlling genes, and PI3K/AKT/PTEN signaling in HK-2 cells; $n=3 .{ }^{* *} P<0.01$ vs. VH; ${ }^{\#} P<0.05$ vs. TAC; ${ }^{\$} P<0.05$ vs. TAC + LC.

stress and programmed cell death through PI3K/AKT/PTEN signaling may account for these renoprotective effects of LC. Our findings provide a potential rationale for the use of a conventional drug to prevent immunosuppressant-related complications.

\section{ACKNOWLEDGEMENTS}

This work was supported by the National Natural Science Foundation of China (No. $81560125,81760293,81760132$, and 81760668).

\section{AUTHOR CONTRIBUTIONS}

$\mathrm{CL}, \mathrm{CWY}, \mathrm{JPL}, \mathrm{BHC}$, and BSC designed the research; YJJ, HLZ, JJ, and SGP performed the research; HYL, HYZ, and CLZ conducted the in vitro study; MYX, YSJ, SC, and JZJ analyzed the data and performed molecular work; and $\mathrm{CL}$ wrote the paper.

\section{ADDITIONAL INFORMATION}

Competing interests: The authors declare no competing interests.

\section{REFERENCES}

1. Bentata Y. Tacrolimus: 20 years of use in adult kidney transplantation. What we should know about its nephrotoxicity. Artif Organs. 2020;44:140-52.

2. Yap DY, Ma MK, Mok MM, Kwan LP, Chan GC, Chan TM. Long-term data on tacrolimus treatment in lupus nephritis. Rheumatology. 2014;53:2232-7.

3. Sinha A, Sharma A, Mehta A, Gupta R, Gulati A, Hari P, et al. Calcineurin inhibitor induced nephrotoxicity in steroid resistant nephrotic syndrome. Indian J Nephrol. 2013;23:41-6.

4. Yagisawa T, Omoto K, Shimizu T, Ishida H, Tanabe K. Arteriosclerosis in zero-time biopsy is a risk factor for tacrolimus-induced chronic nephrotoxicity. Nephrology. 2015:20 Suppl 2:51-7.
5. Lim SW, Shin YJ, Luo K, Quan Y, Ko EJ, Chung BH, et al. Effect of klotho on autophagy clearance in tacrolimus-induced renal injury. FASEB J. 2019;33:2694-706.

6. Zhang LY, Jin J, Luo K, Piao SG, Zheng HL, Jin JZ, et al. Shen-Kang protects against tacrolimus-induced renal injury. Korean J Intern Med. 2019;34:1078-90.

7. Ferreira GC, McKenna MC. L-carnitine and acetyl-L-carnitine roles and neuroprotection in developing brain. Neurochem Res. 2017;42:1661-75.

8. Giudetti AM, Stanca E, Siculella L, Gnoni GV, Damiano F. Nutritional and hormonal regulation of citrate and carnitine/acylcarnitine transporters: two mitochondrial carriers involved in fatty acid metabolism. Int J Mol Sci. 2016;17:E817.

9. Xue M, Chen X, Guo Z, Liu X, Bi Y, Yin J, et al. L-carnitine attenuates cardiac dysfunction by ischemic insults through Akt signaling pathway. Toxicol Sci. 2017;160:341-50.

10. Kunak CS, Ugan RA, Cadirci E, Karakus E, Polat B, Un H, et al. Nephroprotective potential of carnitine against glycerol and contrast-induced kidney injury in rats through modulation of oxidative stress, proinflammatory cytokines, and apoptosis. Br J Radio. 2016;89:20140724.

11. Liu Y, Yan S, Ji C, Dai W, Hu W, Zhang W, et al. Metabolomic changes and protective effect of $(L)$-carnitine in rat kidney ischemia/reperfusion injury. Kidney Blood Press Res. 2012;35:373-81.

12. Fan JP, Kim D, Kawachi $H$, Ha TS, Han GD. Ameliorating effects of $L$-carnitine on diabetic podocyte injury. J Med Food. 2010;13:1324-30.

13. Zambrano S, Blanca AJ, Ruiz-Armenta MV, Miguel-Carrasco JL, Arevalo M, Mate A et al. $L$-carnitine attenuates the development of kidney fibrosis in hypertensive rats by upregulating PPAR-gamma. Am J Hypertens. 2014;27:460-70.

14. Boonsanit D, Kanchanapangka S, Buranakarl C. L-carnitine ameliorates doxorubicin-induced nephrotic syndrome in rats. Nephrology. 2006;11:313-20.

15. Xiang Y, Piao SG, Zou HB, Jin J, Fang MR, Lei DM, et al. L-carnitine protects against cyclosporine-induced pancreatic and renal injury in rats. Transplant Proc. 2013; 45:3127-34.

16. Jin J, Jin L, Luo K, Lim SW, Chung BH, Yang CW. Effect of empagliflozin on tacrolimus-induced pancreas islet dysfunction and renal injury. Am J Transplant. 2017;17:2601-16.

17. Yu JH, Lim SW, Luo K, Cui S, Quan Y, Shin YJ, et al. Coenzyme Q10 alleviates tacrolimus-induced mitochondrial dysfunction in kidney. FASEB J. 2019;33: 12288-98. 
18. Lim SW, Shin YJ, Luo K, Quan Y, Cui S, Ko EJ, et al. Ginseng increases klotho expression by FoxO3-mediated manganese superoxide dismutase in a mouse model of tacrolimus-induced renal injury. Aging. 2019;11:5548-69.

19. Wang S, Xu J, Zheng J, Zhang X, Shao J, Zhao L, et al. Anti-inflammatory and antioxidant effects of acetyl-L-carnitine on atherosclerotic rats. Med Sci Monit. 2020;26:e920250.

20. Rababa'h SY, Alzoubi KH, Hammad HM, Alquraan L, El-Salem K. Memory impairment induced by chronic psychosocial stress is prevented by $L$-carnitine. Drug Des Dev Ther. 2019;13:4341-50.

21. Li $M, X u S$, Geng $Y$, Sun $L$, Wang $R$, Yan $Y$, et al. The protective effects of $L$ carnitine on myocardial ischaemia-reperfusion injury in patients with rheumatic valvular heart disease undergoing CPB surgery are associated with the suppression of NF-kappaB pathway and the activation of Nrf2 pathway. Clin Exp Pharmacol Physiol. 2019;46:1001-12.

22. Hong ES, Kim EK, Kang SM, Khang AR, Choi SH, Park KS, et al. Effect of carnitineorotate complex on glucose metabolism and fatty liver: a double-blind, placebocontrolled study. J Gastroenterol Hepatol. 2014;29:1449-57.

23. Mescka CP, Guerreiro G, Donida B, Marchetti D, Wayhs CA, Ribas GS, et al Investigation of inflammatory profile in MSUD patients: benefit of $L$-carnitine supplementation. Metab Brain Dis. 2015;30:1167-74.

24. Jamilian M, Foroozanfard F, Kavossian E, Aghadavod E, Amirani E, Mahdavinia $M$, et al. Carnitine and chromium co-supplementation affects mental health, hormonal, inflammatory, genetic, and oxidative stress parameters in women with polycystic ovary syndrome. J Psychosom Obstet Gynaecol. 2019; $5: 1-9$.

25. Kim YG, Kim SM, Kim KP, Lee SH, Moon JY. The role of inflammasomedependent and inflammasome-independent NLRP3 in the kidney. Cells. 2019, 8:E1389.

26. Liu H, Chen Z, Weng X, Chen H, Du Y, Diao C, et al. Enhancer of zeste homolog 2 modulates oxidative stress-mediated pyroptosis in vitro and in a mouse kidney ischemia-reperfusion injury model. FASEB J. 2020;34:835-52.

27. Chung SD, Lai TY, Chien CT, Yu HJ. Activating Nrf-2 signaling depresses unilateral ureteral obstruction-evoked mitochondrial stress-related autophagy, apoptosis and pyroptosis in kidney. PLoS One. 2012;7:e47299.
28. Peng X, Yang T, Liu G, Liu H, Peng Y, He L. Piperine ameliorated lupus nephritis by targeting AMPK-mediated activation of NLRP3 inflammasome. Int Immunopharmacol. 2018;65:448-57.

29. Wang Y, Zhu X, Yuan S, Wen S, Liu X, Wang C, et al. TLR4/NF-kappaB signaling induces GSDMD-related pyroptosis in tubular cells in diabetic kidney disease. Front Endocrinol. 2019;10:603.

30. Kim SM, Kim YG, Kim DJ, Park SH, Jeong KH, Lee $\mathrm{YH}$, et al. Inflammasomeindependent role of NLRP3 mediates mitochondrial regulation in renal injury. Front Immunol. 2018;9:2563.

31. Shakeri $A$, Tabibi $H$, Hedayati $M$. Effects of $L$-carnitine supplement on serum inflammatory cytokines, c-reactive protein, lipoprotein (a), and oxidative stress in hemodialysis patients with Lp (a) hyperlipoproteinemia. Hemodial Int. 2010;14:498-504.

32. Galvan DL, Green NH, Danesh FR. The hallmarks of mitochondrial dysfunction in chronic kidney disease. Kidney Int. 2017;92:1051-7.

33. Ye J, Li J, Yu Y, Wei Q, Deng W, Yu L. L-carnitine attenuates oxidant injury in HK-2 cells via ROS-mitochondria pathway. Regul Pept. 2010;161:58-66.

34. Nguyen LT, Stangenberg S, Chen $\mathrm{H}$, Al-Odat I, Chan YL, Gosnell ME, et al. Lcarnitine reverses maternal cigarette smoke exposure-induced renal oxidative stress and mitochondrial dysfunction in mouse offspring. Am J Physiol Ren Physiol. 2015;308:F689-96.

35. Luo K, Lim SW, Jin J, Jin L, Gil HW, Im DS, et al. Cilastatin protects against tacrolimus-induced nephrotoxicity via anti-oxidative and anti-apoptotic properties. BMC Nephrol. 2019;20:221.

36. Sue YM, Chou HC, Chang CC, Yang NJ, Chou Y, Juan SH. L-carnitine protects against carboplatin-mediated renal injury: AMPK- and PPARa- dependent inactivation of NFAT3. PLoS One. 2014;9:e104079.

37. Chen W, Ruan R, Zhao S, Ning J, Rao T, Yu W, et al. MicroRNA-205 inhibits the apoptosis of renal tubular epithelial cells via the PTEN/Akt pathway in renal ischemia-reperfusion injury. AmJ Transl Res. 2019;11:7364-75.

38. Higgins DF, Ewart LM, Masterson E, Tennant S, Grebnev G, Prunotto M, et al. BMP7-induced-Pteninhibits Akt and prevents renal fibrosis. Biochim Biophys Acta Mol Basis Dis. 2017;1863:3095-104.

39. Song Y, Liu W, Tang K, Zang J, Li D, Gao H. Mangiferin alleviates renal interstitial fibrosis in streptozotocin-induced diabetic mice through regulating the PTEN/ PI3K/Akt signaling pathway. J Diabetes Res. 2020;2020:9481720. 